\title{
Pairing effects on neutrino transport in low-density stellar matter
}

\author{
S.Burrello, ${ }^{1,2}$ M.Colonna, ${ }^{1}$ and F.Matera ${ }^{3}$ \\ ${ }^{1}$ INFN-LNS, Laboratori Nazionali del Sud, 95123 Catania, Italy \\ ${ }^{2}$ Dipartimento di Fisica e Astronomia, Università di Catania, 95123 Catania, Italy \\ ${ }^{3}$ Dipartimento di Fisica e Astronomia, and Istituto Nazionale di Fisica Nucleare, 50019 Sesto Fiorentino, Firenze, Italy
}

(Dated: May 30, 2022)

\begin{abstract}
We investigate the impact of pairing correlations on neutrino transport in stellar matter. Our analysis is extended to nuclear matter conditions where large density fluctuations develop, associated with the onset of the liquid-vapor phase transition, and clustering phenomena occur. Within a thermodynamical treatment, we show that at moderate temperatures, where pairing effects are still active, the scattering of neutrinos in the nuclear medium is significantly affected by pairing correlations, which increase the neutrino trapping, thus modifying the cooling mechanism, by neutrino emission, of neutron stars.
\end{abstract}

PACS numbers: 26.30.Jk,26.60.Gj,64.10.+h,74.20.Fg

Transport properties of neutrinos play an essential role in the description of gravitational collapse, supernovae, protoneutron stars and binary mergers of compact objects. For instance, the cooling process of newly formed neutron stars is, over a broad time interval $\left(10^{-10}-10^{5} \mathrm{yr}\right)$, dominated by the emission of neutrinos of all flavors. In fact, the neutrino flux is the only direct probe of the mechanism of supernovae and the structure of protoneutron stars [1-5].

The most important ingredient of neutrino transport calculations is the neutrino opacity, essentially determined by charged current absorption and neutral current scattering reactions. While scattering from electrons dominates the neutrino thermalization process, neutrino-baryon scattering and absorption are the leading mechanisms for energy and lepton number transport [6-9].

Recently, many efforts have been devoted to describe the neutrino production and interactions in great detail [10-13]. In particular, it appears that this mechanism is clearly affected by general phenomena, such as phase transitions, characterizing the behavior of interacting many-body systems. Liquidgas phase transitions are expected to appear for nuclear matter at sub-saturation density and relatively low temperature $(T \lesssim 15 \mathrm{MeV}$ ), driven by the unstable nuclear mean-field (i.e. by spinodal instabilities) [14]. This important feature of nuclear matter is closely connected to the multifragmentation mechanism experimentally observed in nuclear reactions [15] and to the occurrence of clustering phenomena in the inner crust of neutron stars [16, 17]. It was recently pointed out that large density fluctuations, associated with the first order nuclear liquid-gas phase transition, hugely increase the scattering of neutrinos [18], thus quenching their emissivity processes in low density regions.

As a quite general feature, fermionic systems may also exhibit pairing correlations. Their importance is widely discussed in the astrophysical context, as far as cooling processes [19] and glitch phenomena [20, 21] are concerned.

Since pairing correlations are mostly active at low density and relatively low temperature, below the critical temperature for the transition from superfluid to normal matter, in a certain region of the nuclear matter phase diagram volume instabilities may co-exist with strong pairing effects [22].

The aim of this work is to investigate the influence of pair- ing correlations on the neutrino scattering cross section, in conditions of moderate temperature, charge asymmetry and low density (close to the spinodal border), which are encountered in neutron stars, as well as in supernova explosions (prebounce phase).

At the onset of spinodal instabilities, the speed of sound vanishes and the nucleonic free energy density exhibits a negative curvature, both in symmetric and asymmetric matter. Owing to the global isoscalar-like character of the instability, the density response function is enhanced, thus modifying the neutral current neutrino opacity, whereas charge current absorption processes are not affected. Hence in the following we will concentrate only on neutral current neutrino scattering. We will consider nonrelativistic nucleons coupled to neutrinos through only the vector neutral current, neglecting contributions from the axial current, as spin-density fluctuations do not present any singular behavior for the assumed physical conditions.

Then the differential cross section (per unit of volume $V$ ) for scattering in the medium of neutrinos with energy $E_{v}$, as a function of the neutrino final energy $E_{v}^{\prime}$ and scattering angle $\theta$, is given by [8, 23]:

$$
\frac{1}{V} \frac{d^{3} \sigma}{d E_{v}^{\prime} d \Omega^{2}}=\frac{G_{F}^{2}}{8 \pi^{3}}\left(E_{v}^{\prime}\right)^{2}(1+\cos \theta) S_{V}^{00}(\omega, \mathbf{q}),
$$

where $\omega=E_{v}-E_{v}^{\prime}$ denotes the energy transfer to the medium, $\mathbf{q}$ is the momentum transfer, related to $\omega$ and to the neutrino scattering angle $\theta$ (we use units such that $\hbar=c=k_{B}=1$ ). $G_{F}$ denotes the weak coupling constant and $S_{V}^{00}$ identifies the dynamic form factor, which can be expressed in terms of the nucleon density-density correlation factor as:

$$
S_{V}^{00}(\omega, \mathbf{q})=\int d t d \mathbf{r} e^{i \omega t} e^{-i \mathbf{q} \cdot \mathbf{r}}\left\langle J^{(N) 0}(t, \mathbf{r}) J^{(N) 0}(0,0)\right\rangle,
$$

where $J_{0}^{(N)}(t, \mathbf{r})=\sum_{i=n, p} c_{V}^{(i)} \rho_{i}(t, \mathbf{r})$, with $c_{V}^{(n)}=-0.5, c_{V}^{(p)}=$ 0.036. $\rho_{i}(t, \mathbf{r})(\mathrm{i}=\mathrm{n}, \mathrm{p})$ is the nucleon (neutron or proton) local density. Based on energy conservation, we see that a typical energy transfer $\omega$ from the medium to the neutrino is of the order of $q$ times the thermal velocity of nucleons $(T / m)^{1 / 2}$. Thus, in the limit of heavy nucleons, when we integrate the 
differential cross section over a range of $\omega$ values, the other factors in the integrand can be evaluated at $\omega=0$, so that:

$$
S_{V}^{00}(\omega, \mathbf{q}) \rightarrow \delta(\omega) \int d \omega^{\prime} S_{V}^{00}\left(\omega^{\prime}, \mathbf{q}\right)=2 \pi \delta(\omega) S_{V}^{00}(\mathbf{q})
$$

where $S_{V}^{00}(\mathbf{q})$ is the static structure factor, which, according to Eq.(2), corresponds to $n-n, p-p$ and $n-p$ density fluctuation correlations taken at equal time:

$$
S_{V}^{00}(\mathbf{q})=\left\langle\delta J^{(N) 0}(\mathbf{q}) \delta J^{(N) 0}(-\mathbf{q})\right\rangle .
$$

Exploiting the fluctuation-dissipation theorem [24] and neglecting quantum fluctuations, the static structure factor can be expressed, for a system at temperature $\mathrm{T}$, as:

$S_{V}^{00}(\mathbf{q})=T\left[c_{V}^{(n)^{2}} \mathscr{C}_{n n}^{-1}(q)+c_{V}^{(p)^{2}} \mathscr{C}_{p p}^{-1}(q)+2 c_{V}^{(n)} c_{V}^{(p)} \mathscr{C}_{n p}^{-1}(q)\right]$,

where the matrix $\mathscr{C}^{-1}$ is the inverse of curvature matrix of the system free energy density [8].

In this paper, we consider stellar matter where the proton charge is globally neutralized by a Fermi gas of electrons. The local energy density, which is a function of the total density $\rho=\rho_{n}+\rho_{p}$, the proton fraction $y_{p}=\rho_{p} / \rho$ and the electron density $\rho_{e}$, can be written as:

$$
\mathscr{E}_{\text {tot }}\left(\rho, y_{p}, \rho_{e}\right)=\mathscr{E}_{N M}+\mathscr{E}_{N M}^{f}+\mathscr{E}_{C o u l}+\mathscr{E}_{e}\left(\rho_{e}\right),
$$

where $\mathscr{E}_{e}$ is the energy density associated with the electron kinetic energy and the contributions of the Coulomb term, $\mathscr{E}_{\text {Coul }}$, related to the interaction between all charges (protons and electrons), and of nuclear matter surface terms, $\mathscr{E}_{N M}^{f}$, are explicitely evidenced.

The electron term is readily evaluated in the approximation of a degenerate, ultrarelativistic Fermi gas, hence the electron chemical potential, $\mu_{e}$, is just equal to the electron Fermi momentum.

The spin-saturated nuclear matter energy density, $\mathscr{E}_{N M}$, at finite temperature $\mathrm{T}$, in the BCS approximation reads [22, 25]:

$$
\mathscr{E}_{N M}\left(\rho, y_{p}\right)=\sum_{i=n, p}\left[2 \int \frac{d \mathbf{p}}{(2 \pi)^{3}} f_{i} \frac{p^{2}}{2 m_{i}^{*}}+\frac{1}{4} v_{\pi}\left(\rho_{i}\right)\left|\tilde{\rho}_{i}\right|^{2}\right]+\mathscr{E}_{s k y}
$$

In the previous equation, $f_{i}$ is the occupation number for a nucleon of species $i$ with momentum $\mathbf{p}$ and $\tilde{\rho}_{i}=2 \Delta_{i}\left(\rho_{i}\right) / v_{\pi}\left(\rho_{i}\right)$ denotes the anomalous density with the temperature dependent pairing gap $\Delta_{i}$. The corresponding quasiparticle energies are given by $E_{\Delta}=\sqrt{\xi^{2}+\Delta_{i}^{2}}$, where $\xi=p^{2} / 2 m_{i}^{*}-\mu_{i}+U_{i}$, being $\mu_{i}$ and $U_{i}=\frac{\partial \mathscr{E}_{N M}}{\partial \rho_{i}}$ the chemical and mean field potential, respectively, for each nucleonic species $i$. We note that, in the definition of the mean-field potential $U_{i}$, the derivative with respect to $\rho_{i}$ is taken at constant $\tilde{\rho}_{i}$.

In the following numerical applications, we will use the SAMi-J35 parametrization [26] of the Skyrme energy functional for the local energy density $\mathscr{E}_{s k y}$ and the effective nucleon mass $m_{i}^{*}$. For the pairing term, we adopt here the same interaction as in [22, 25], whose density dependent strength,

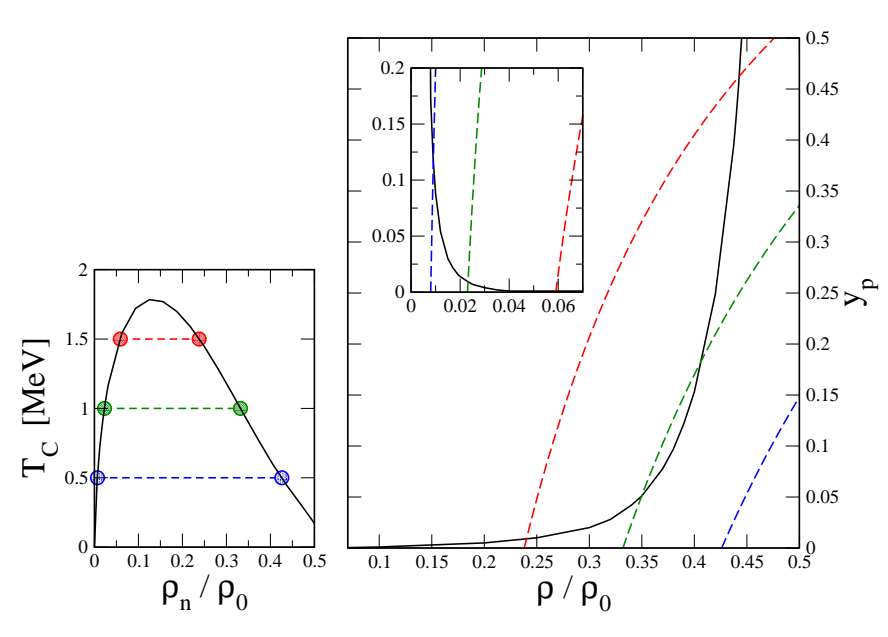

FIG. 1: (Color online) Left panel: The critical temperature for the transition from superfluid to normal matter, as a function of the reduced neutron density $\rho_{n} / \rho_{0}$. Right panel: The spinodal border (full line), in the $\left(\rho, y_{p}\right)$ plane, associated with temperature $\mathrm{T}=0.5 \mathrm{MeV}$ and momentum transfer $q=30 \mathrm{MeV}$. The inset shows a zoom of the low-density region. The dashed lines are curves of constant neutron density, corresponding to the values associated with the circles in the left panel (see text for more details).

$v_{\pi}\left(\rho_{i}\right)$, is calculated exactly in the BCS approximation by inverting the gap equation, to reproduce the ${ }^{1} S_{0}$ pairing gap of pure neutron matter given by Brueckner-Hartree-Fock calculations [27]. It should be remarked that, according to the asymmetry conditions of the stellar matter, the pairing interaction acts only between nucleons of the same type. The results are then extended to the $p p$ case, assuming that the pairing strength is the same as in the $n n$ case, just depending on the density of the species considered. Within this framework, one can then determine the derivatives of $\mu_{i}$ with respect to $\rho_{i}$ and so evaluate the curvature matrix [28], which is needed in the calculation of the cross section:

$$
\begin{gathered}
\mathscr{C}(q)=\left(\begin{array}{ccc}
\partial_{\rho_{n}} \mu_{n} & \partial_{\rho_{p}} \mu_{n} & 0 \\
\partial_{\rho_{n}} \mu_{p} & \partial_{\rho_{p}} \mu_{p} & 0 \\
0 & 0 & \partial_{\rho_{e}} \mu_{e}
\end{array}\right)+2 q^{2}\left(\begin{array}{ccc}
C_{n n}^{f} & C_{n p}^{f} & 0 \\
C_{p n}^{f} & C_{p p}^{f} & 0 \\
0 & 0 & 0
\end{array}\right) \\
+\frac{4 \pi e^{2}}{q^{2}}\left(\begin{array}{ccc}
0 & 0 & 0 \\
0 & 1 & -1 \\
0 & -1 & 1
\end{array}\right)
\end{gathered}
$$

where $e^{2}=1.44(\mathrm{MeV} \cdot \mathrm{fm})$ and the coefficients $C_{i j}^{f}$ are combinations of the Skyrme surface parameters [28].

The pairing interaction modifies neutron and proton chemical potentials, and their derivatives, which appear in the curvature matrix, Eq. (8). Thus, in suitable conditions of density, asymmetry and temperature, we can expect a non-negligible impact on the neutrino differential cross section.

As stressed before, neutrino trapping is quite influenced by large density fluctuations of the nuclear density, which develop close to the spinodal border and may lead to clustering 


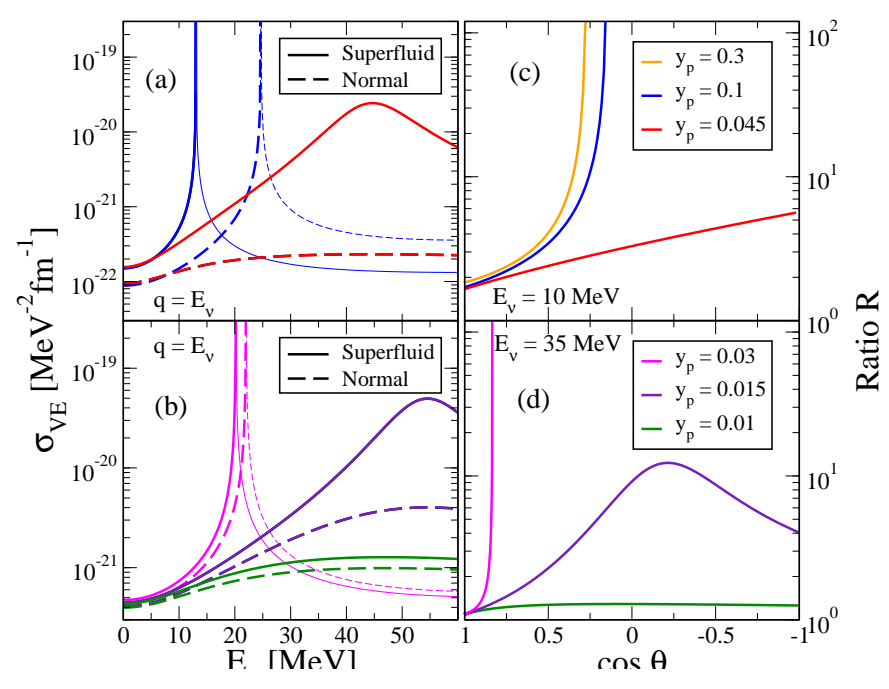

FIG. 2: (Color online) Left panels: Neutrino differential cross sections, $\sigma_{V E}$ (see text), as a function of the neutrino energy, obtained in the full calculation (full lines) or neglecting the pairing interaction (dashed lines). Right panels: Ratio $R$ between the full calculation and the results obtained neglecting the pairing interaction, as a function of the cosine of the neutrino scattering angle $\theta$, for selected neutrino energies. Results are shown for the following conditions: $\rho=\rho_{0} / 100-\mathrm{T}=0.5 \mathrm{MeV}$ (panels (a) and (c)) and $\rho=\rho_{0} / 4-\mathrm{T}=$ 1.4 MeV (panels (b) and (d)). The proton fractions considered are indicated inside the figure.

phenomena. Within the framework adopted here (see Eq.(5)), the amplitude of neutron and proton density fluctuations is essentially related to the inverse of the eigenvalues of the curvature matrix and so become quite large when the isoscalar-like one, $\lambda_{S}(q)$, is small. In this case, we expect pairing correlations to have a large relative weight on the curvature matrix elements, especially close to the critical temperature, $T_{c}$, for the transition from normal to superfluid matter, where discontinuities appear in the chemical potential derivatives [22]. Hence we will discuss stellar matter at moderate temperature (below $2 \mathrm{MeV}$ ), at density and asymmetry conditions close to the spinodal border, where $\lambda_{S}$ vanishes.

For the sake of simplicity, let us start considering only neutron pairing. As shown in the following, the latter leads, in any case, to the largest effects. In Fig. 1 (left panel) we represent the critical temperature, $T_{c}$ as a function of the neutron density. The right panel shows the spinodal border (full line), in the $\left(\rho, y_{p}\right)$ plane, at a temperature and $q$ values of interest for our study. It should be noticed that, because of Coulomb and surface effects, the spinodal border depends on $q$, but it is not very sensitive to the temperature, within the range considered in our study. For a fixed value of the temperature $\mathrm{T}$, the plot on the left panel allows one to identify two values of the neutron density $\rho_{n}$ (see the circles) for which the temperature considered corresponds to the critical one, so pairing effects are expected to be large. Each value of the neutron density defines an hyperbole in the $\left(\rho, y_{p}\right)$ plane (see the dashed lines on the right panel). Then the crossing of the dashed lines with the spinodal border identifies the density-asymmetry regions

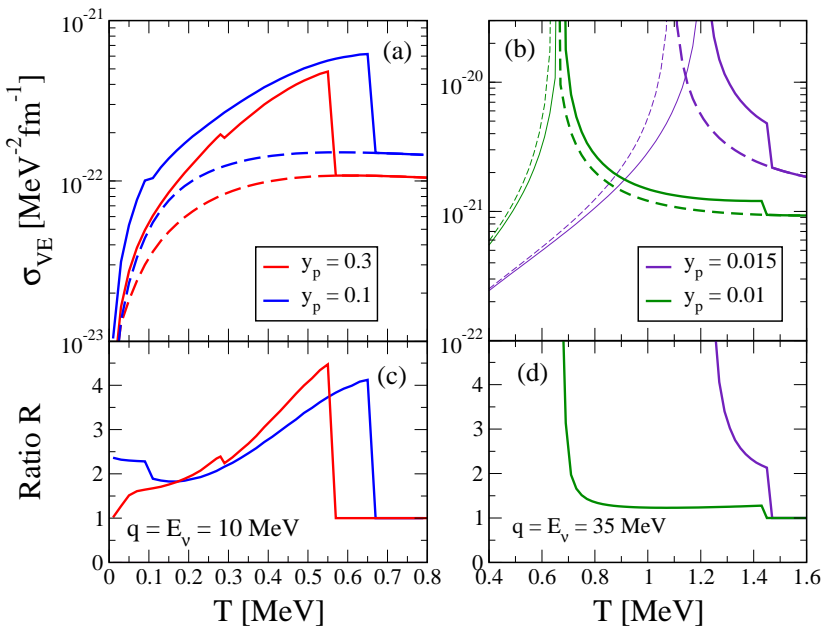

FIG. 3: (Color online) Top panels: Neutrino differential cross section, $\sigma_{V E}$ (see text), as a function of the temperature $\mathrm{T}$, obtained in the full calculation (full lines) or neglecting the pairing interaction (dashed lines). Bottom panels: Ratio $R$ between the full calculation and the results obtained neglecting the pairing interaction, as a function of the temperature T. Results are shown for the following conditions: $\rho=\rho_{0} / 100$ (panels (a) and (c)) and $\rho=\rho_{0} / 4$ (panels (b) and (d)). The proton fractions considered are indicated inside the figure.

where large density fluctuations can coexist with important pairing contributions. It appears that a variety of conditions, from very small densities up to $\rho \approx 0.4 \rho_{0}$ (being $\rho_{0}=0.16$ $\mathrm{fm}^{-3}$ the saturation density) and with proton fraction ranging from quite low values up to $y_{p} \approx 0.5$, are good candidates for our study. These conditions may occur in the inner crust of a neutron star or in the pre-bounce phase of a supernova explosion, when the temperature is still low (see e.g. refs. [3, 29. 32]).

Guided by this analysis, in the following we will discuss the results obtained for the two sets of parameters: (1) $\rho=$ $\rho_{0} / 100$, at $\mathrm{T}=0.5 \mathrm{MeV}$, and (2) $\rho=\rho_{0} / 4$, at $\mathrm{T}=1.4 \mathrm{MeV}$, i.e. we will consider two opposite density regimes at temperatures where we expect large pairing effects. Several $y_{p}$ values will be tested, close to the values suggested by Fig.1. For the conditions considered now, only neutrons are paired, because the temperature values are always above the proton critical temperature.

In Fig.2 (left panel) we display the neutrino differential cross section, $\sigma_{V E} \equiv 1 /\left(V E_{v}^{2}\right) d^{2} \sigma / d \Omega^{2}$ (full lines), as a function of the neutrino energy $E_{V}$ (we consider momentum transfer $q=E_{v}$ ). To underline the importance of pairing effects, we also show (dashed lines) the results obtained neglecting the pairing interaction in our calculations. At the lowest density considered (case (1), panel (a)), the proton fraction $y_{p}=0.045$ corresponds to conditions which are close, but outside the spinodal region for all $q$ values. One can notice quite large pairing effects on the cross section, especially for intermediate neutrino energies, where a bump is observed in the full calculation. In this case the momentum transfer corresponds to density oscillations not much affected by Coulomb (act- 
ing at small $q \mathrm{~s}$ ) or surface (acting at large $q \mathrm{~s}$ ) effects, so that $\lambda_{S}$ remains close to zero for an appreciable extent of the momentum transfer and the pairing effects are clearly enhanced. We notice that pairing correlations go in the direction of reducing the curvature of the free energy density, leading to an increase of density fluctuations and related neutrino cross section. This effect is associated with neutron pairing and indicates that neutron correlations favours matter clustering.

Due to Coulomb repulsion effects, when the momentum transfer $q$ approaches zero, the eigenvalue $\lambda_{S}(q)$ is always positive and therefore density oscillations are stable. However, for higher proton fractions (see the result for $y_{p}=0.1$ ), nuclear matter approaches the spinodal border already for oscillations related to quite low momentum transfer. At the crossing, a divergent behavior is observed for density fluctuations and neutrino cross section. This is reminiscent of the well-known critical-opalescence phenomenon observed in the light scattering through a fluid near the critical temperature of the liquid-vapor transition [33]. Clearly here one should go beyond the second curvature of the free energy density, in order to evaluate density fluctuations accurately. However, our calculations already point out strong pairing effects, with a significant shift to smaller values of the neutrino energy associated with the divergency in the full calculations. This implies that also less energetic neutrinos have more chances to be trapped, so that the energy flux carried away by neutrinos could be damped by pairing correlations.

Larger transfer momenta $q$ s correspond to unstable oscillations. In this case $\lambda_{S}(q)$ is negative and one cannot simply apply the prescription given above (Eq.(7)) to evaluate neutron and proton density fluctuations. Indeed, whereas in stable situations the variance associated with isoscalar-like fluctuations equals $\sigma_{S}(q)=T / \lambda_{S}(q)$, in presence of instabilities it grows exponentially with time until a new equilibrium condition, corresponding to clustered matter, is reached [14]. Hence, inside the spinodal region the correct equilibrium fluctuations cannot be estimated within our framework. However, as a first order approximation, we assume that the equilibrium variance is close to the value obtained, for each $q$, at the time $t \approx \tau(q)$, being $\tau(q)$ the instability growth time of the $q$ mode considered [34], leading to $\sigma_{S}(q) \approx T /\left|\lambda_{S}\right|$. Then the elements of the curvature matrix, Eq.(7), are modified accordingly. The corresponding neutrino cross sections are indicated by thin lines in Fig.2.

The panel (b) displays the results obtained for case (2), where, as indicated by the analysis shown in Fig.1, we take smaller proton fractions $y_{p}$. We note that this also corresponds to the trend predicted for the proton fraction in the inner crust of neutron stars [17, 32]. The same considerations made above for the lower density case hold. However here pairing effects, though still quite significant, are reduced with respect to the previous case, just because they are linked to the derivative of the pairing gap (and thus of the critical temperature) with respect to the density [22], which is steeper for case (1) (see Fig. 1).

To emphasize the role of pairing effects, panels (c),(d) of Fig 2 represent the ratio $R$ between the cross section associ- ated with the full calculations and the results obtained neglecting the pairing interaction, as a function of $\cos \theta$, for selected neutrino energies, representative of $\beta$-equilibrium conditions. We note that the neutrino scattering angle is related to the momentum transfer $q$. Unstable $q$ values are not considered in this plot. Results are shown for three proton fractions, for cases (1) and (2). From this representation, it clearly emerges how huge pairing effects become approaching the spinodal border.

The influence of the temperature on our results is discussed in Fig. 3, where the quantity $\sigma_{V E}$ is displayed, for selected neutrino energies and $q=E_{v}$, as a function of $\mathrm{T}$, for density conditions as in case (1) (panel (a)) and case (2) (panel (b)). Two proton fractions are considered. The full calculations are compared to those obtained neglecting the pairing interaction. The momentum transfer considered in panel (a) corresponds to stable oscillations. Pairing effects are quite important already at very low temperature, where $p p$ pairing is also present, but they increase approaching the neutron critical temperature, $T_{c} \approx 0.65(0.55) \mathrm{MeV}$ for $y_{p}=0.1(0.3)$, and then vanish. Indeed, quite interestingly, we observe a jump in the cross section at $T=T_{c}$, which suddenly reaches the value of normal nuclear matter. A small jump also occurs, for both proton fractions, at a lower temperature, due to the disappearance of proton pairing. The jumps observed are related to the discontinuity emerging in the density derivative of the chemical potential, $\partial \mu_{i} / \partial \rho_{i}$, which is connected to the matter compressibility, in analogy with the well known heat capacity discontinuity [22, 32].

The conditions of panel (b) of Fig 3 are such that the $q$ value considered corresponds to fluctuations which are unstable at zero temperature. A divergency occurs for the cross sections at the temperature associated with the crossing of the spinodal border. Then, at higher temperature, density oscillations become stable. We note that, inspite of the increasing temperature, the cross section reduces when the nuclear matter moves away from the spinodal border. Also in this case a discontinuity is observed at the neutron critical temperature. To better evidence the role of pairing effects, panels (c) and (d) of Fig. 3 show the ratio $R$ between the full calculations and the results obtained neglecting pairing correlations.

To conclude, our analysis evidences important pairing effects on neutrino transport, for suitable density, asymmetry and temperature conditions, which are of relevant interest for the evolution of neutron stars and supernovae explosion in the pre-bounce phase [31, 32]. We concentrate on the behavior of low-density matter, close to the spinodal border, characterized by quite large density fluctuations. Focusing on neutral current neutrino scattering, we generally observe an increase of the neutrino differential cross section in paired matter, thus enhancing neutrino trapping and reducing the energy flux carried out by neutrino emission. This is essentially due to attractive neutron-neutron pairing correlations, which favour lowdensity clustering. From this study new hints emerge about a significant impact of pairing effects on the cooling mechanism, by neutrino emission, of low-density stellar matter at moderate temperature. 
[1] H. A. Bethe and J. R. Wilson, Astrophys. J. 295, 14 (1985).

[2] A. Burrows, Annu. Rev. Nucl. Part. Sci. 40, 181 (1990).

[3] M. Prakash, J. M. Lattimer, J. A. Pons, A. W. Steiner, and S. Reddy, in Lectures Notes in Physics, edited by D. Blaschke, N. K. Glendenning, and A. Sedrakian (Springer-Verlag, Berlin, 2001) Vol. 578, p. 364.

[4] H. T. Janka, K. Langanke, A. Marek, G. Martínez-Pinedo, and B. Mueller, Phys. Rep. 442, 38 (2007).

[5] M. Baldo et al., Phys. Rev. C 89, 048801 (2014).

[6] S. Reddy, M. Prakash, and J. M. Lattimer, Phys. Rev. D 58, 013009 (1998).

[7] S. Reddy, M. Prakash, J. M. Lattimer, and J. A. Pons, Phys. Rev. C 59, 2888 (1999).

[8] A. Burrows and R. F. Sawyer, Phys. Rev. C 58, 554 (1998).

[9] S. Reddy, G. F. Bertsch, and M. Prakash, Phys. Lett. B 475, 1 (2000).

[10] G. Watanabe, K. Sato, K. Yasuoka, and T. Ebisuzaki. Phys. Rev. C 68, 035806 (2003).

[11] C. J. Horowitz, M. A. Pérez-García, and J. Piekarewicz, Phys. Rev. C 69, 045804 (2004).

[12] G. Martínez-Pinedo, T. Fischer, A. Lohs, and L. Huther, Phys. Rev. Lett. 109, 251104 (2012).

[13] H. Pais, W. G. Newton, and J. R. Stone, Phys. Rev. C 90, 065802 (2014).

[14] Ph. Chomaz, M. Colonna, and J. Randrup, Phys. Rep. 389, 263 (2004).

[15] B. Borderie and M. F. Rivet, Progr. in Part. and Nucl. Phys. 61 (Book Series), 551 (2008), and refs. therein.

[16] J. M. Lattimer and M. Prakash, Phys. Rep. 442, 109 (2007); A. W. Steiner, J. M.Lattimer, and E. F. Brown, Astrophysical J. 722, 33 (2010).

[17] Ad. R. Raduta, F. Gulminelli, and F. Aymard, Eur. Phys. J. A50, 24 (2014).
[18] J. Margueron, J. Navarro, and P. Blottiau, Phys. Rev. C 70, 028801 (2004).

[19] D.Page, J. M. Lattimer, M. Prakash, and A. W. Steiner, Astrophys. J. 707, 1131 (2009).

[20] B. Carter, in Lectures Notes in Physics, edited by D. Blaschke, N. K. Glendenning, and A. Sedrakian (Springer-Verlag, Berlin, 2001) Vol. 578, p. 54.

[21] A. Sedrakian, Phys. Rev. D 71, 083003 (2005).

[22] S. Burrello, M. Colonna, and F. Matera, Phys. Rev. C 89, 057604 (2014).

[23] N. Iwamoto and C. J. Pethick, Phys. Rev. D 25, 313 (1982).

[24] L. D. Landau and E. M. Lifshitz, Statistical Physics Part 1, Vol. 5 (3 ed.) (Butterworth-Heinemann, Oxford, 1980).

[25] N. Chamel, Phys. Rev. C 82, 014313 (2010).

[26] X. Roca-Maza et al., Phys. Rev. C 87, 034301 (2013).

[27] L. G. Cao, U. Lombardo, and P. Schuck , Phys. Rev. C 74, 064301 (2006).

[28] C. Ducoin, Ph. Chomaz, and F. Gulminelli, Nucl. Phys. A 789, 403 (2007).

[29] R. Mayle, J. R. Wilson, and D. N. Schramm, Astrophys. J. 318, 288 (1987).

[30] D. Page and S. Reddy, in Neutron Star Crust, edited by C. A. Bertulani and J. Piekarewicz (Nova Science Publishers, New York, 2012), p. 281.

[31] N. Buyukcizmeci et al., Nucl. Phys. A 907, 13 (2013).

[32] S. Burrello et al., Phys. Rev. C 92, 055804 (2015).

[33] L. D. Landau, E. M. Lifshitz, and L. P. Pitaevskii, Electrodynamics of Continuous Media, Vol. 8 (2 ed.) (ButterworthHeinemann, Oxford, 1984).

[34] M. Colonna and F. Matera, Phys. Rev. C 71, 064605 (2005); Phys. Rev. C 77, 064606 (2008). 